Z. klin. Chem. u. klin. Biochem.

8. Jg., S. 605-610, November 1970

\title{
Gesamtthyroxin im Serum
}

(Vergleichsuntersuchungen mit drei Methoden)

\author{
Von J. Herrmann' ${ }^{1}$ ), A. Brase 2 ), H. de Marées ${ }^{3}$ ) und H. L. KrüsKemper ${ }^{1}$ )
}

Medizinische Hochschule Hannover

(Eingegangen am 23. 7. 1970)

\begin{abstract}
3 Methoden zur Bestimmung des Gesamtthyroxins im Serum werden hinsichtlich Richtigkeit und Präzision miteinander verglichen. Die praktische Bewährung der drei Tests wurde anhand von 179 Seren überprüft.

Die Errechnung des Gesamt-T $T_{4}$ aus dem $P B^{127} I\left(T_{4}-P B I\right)$ ergab sowohl „,in Serie“ als auch „von Tag zu Tag“ mit Variationskoeffizienten von 3,5 bzw. 3,1\% die beste Präzision. Auch bei der Prüfung der Richtigkeit zeigte sich für dás $\mathrm{T}_{4}$-PBI die beste Korrelation zwischen Beobachtung und Erwartung $(r=0,9972)$. Der Nachteil der Methode liegt in der fehlenden Spezifität für Thyroxin.

Bei der direkten Bestimmung des Gesamtthyroxins nach der Isotopenverdiunnungsmethode von MurPHY und PATTEE ( $\left.T_{4}-M\right)$ lag der Variationskoeffizient als $\mathrm{Maß}$ der Präzision doppelt so hoch wie beim $T_{4}$-PBI. Die Richtigkeit erfüllte mit einem $r=0,989$ die üblichen Anforderungen an ein klinisch-chemisches Verfahren. Vorteil des Isotopenverdünnungsprinzips ist seine absolute Spezifität für Thyroxin. Die Bestimmungskapazität entspricht mit 100 Seren/5 Stdn. etwa der Leistungsfähigkeit der automatischen PBI-Bestimmung.

Eine kommerzielle Modifikation der Murphy-PAtreE-Methode (Tetrasorb-Abbott-Test; $T_{4}-T$ ) erwies sich sowohl hinsichtlich der Pràzision $(\mathrm{VK}=15,8$ bzw. 11,6\%) als auch der Richtigkeit $(r=0,963)$ als den beiden anderen Verfahren unterlegen. Besonders im Meßbereich über $12 \mu \mathrm{g} / 100 \mathrm{~m} l$ Thyroxin waren Präzision und Genauigkeit unbefriedigend.
\end{abstract}

\section{The measurement of the total thyroxin in serum (a comparison of three methods)}

Three methods for the determination of total serum thyroxin were compared with respect to accuracy and precision in trials involving 179 sera.

The calculation of total $T_{4}$ from the $P B^{127} I\left(T_{4}-P B I\right)$ gave the best precision with variation coefficients of 3.5 and $3.1 \%$ "in series" and from "day to day" respectively. In the test of accuracy, the $\mathrm{T}_{\mathbf{4}}-\mathrm{PBI}$ also gave the best correlation between observed and expected $(\mathrm{r}=0.9972)$. The disadvantage of the method is its lack of specificity for thyroxin.

In the direct determination of total thyroxin by the isotope dilution method of MuRpHY and PATTEE $\left(T_{4}-M\right)$, the variation coefficient, as a measure of precision, was twice as high as for $\mathrm{T}_{4}$-PBI. The accuracy, with $\mathrm{r}=0.989$, satisfied the normal requirements of a clinical chemical method. The advantage of the isotope dilution method is its absolute specificity for thyroxin. The through-put is $100 \mathrm{sera} / 5 \mathrm{hr}$, which is about the same as for the automatic PBI determination.

A commercial modification of the MURPHY-PATteE method (Tetrasorb-Abbott-Test; $T_{4}-T$ ) was inferior to the other two methods both in precision $(V C=15.8$ and $11.6 \%)$ and accuracy $(\mathrm{r}=0.963)$. Precision and accuracy were especially unsatisfactory above $12 \mu \mathrm{g} / 100 \mathrm{~m} /$ thyroxin.

Die Bestimmung der Schilddrüsenhormonkonzentration im Serum wird als die wertvollste labortechnische Hilfe angesehen, Aufschlüsse über die Funktion der Schilddrüse zu erlangen. Seit langer Zeit hat die Bestimmung des proteingebundenen Jods $\left.\left(\mathrm{PB}^{127} \mathrm{I}\right)^{4}\right)$ in dieser Hinsicht einen festen Platz. Dạüber hinaus ist es neuerdings möglich, die mangelnde Spezifität der Hormonjodbestimmung für Schilddrüsenhormon nach Entwicklung einer für Thyroxin spezifischen Methode zu umgehen; das zuerst von EKINS (1) als Sättigungsanalyse beschriebene und auf dem Prinzip der Radioisotopenverdrängung beruhende Verfahren wurde vor allem von Murphy und Pattee $(2,3)$ so weit vervollkommnet und vereinfacht, $\mathrm{da} ß$ Routinebestimmungen in ausreichender Zahl möglich wurden. Seit kurzem werden kommer-

1) Abt. Klin. Endokrinologie, Dept. Innere Medizin.

2) Institut für Nuklearmedizin, Radiologisches Zentrum.

3) Department Physiologie.

4) Abkürzungen: $\mathrm{PB}^{127} \mathrm{I}=$ proteingebundenes ${ }^{127} \mathrm{Jod} ; \mathrm{T}_{4}=$ Thyroxin; TBG $=$ Thyroxin-bindendes Globulin; $\mathrm{PB}^{131} \mathrm{I}=$ proteingebundenes ${ }^{131} \mathrm{Jod} ; \mathrm{T}_{3}=$ Trijodthyronin. zielle, fertige Testsätze (z. B. Tetrasorb-Аввотт) für die Messung des Gesamtthyroxins angeboten, die ebenfalls auf dem Prinzip der Isotopenverdrängung beruhen, sich aber in der Durchführung der Bestimmung von der Originalmethode von MURphy und PatTeE (3) unterscheiden.

Ziel der vorliegenden Arbeit ist es, die mit 3 Methoden

1. Gesamt- $\mathrm{T}_{4}{ }^{4}$ ) aus dem $\mathrm{PB}^{127} \mathrm{I}$ errechnet $\left(\mathrm{T}_{4}-\mathrm{PBI}\right)$;

2. Gesamt- $T_{4}$ nach Murphy und Pattee $\left(\mathrm{T}_{4}-\mathrm{M}\right)$;

3. Gesamt- $T_{4}$ nach Tetrasorb-Test $\left(\mathrm{T}_{4}-\mathrm{T}\right)$

gewonnenen Ergebnisse hinsichtlich Richtigkeit und Präzision zu vergleichen, und die praktische Bewährung der drei Teste anhand von 179 Seren (131 Euthyreosen, 33 Hyperthyreosen, 15 Hypothyreosen) zu überprüfen.

\section{Methoden und Material}

Das $P B^{127} I$ wurde mit der AutoAnalyzcr-Methode unter Verwendung von Amberlite-IRA $401(200 \mathrm{mg} / 2 \mathrm{ml})$ als Ionenaustauscherharz gemessen. 
Das $T_{4}-P B I$ wurde aus dem PBI-Wert durch Multiplikation mit dem Faktor 1,53 errechnet.

Das $T_{4}-M$ wurde nach der Vorschrift von Murphy und Pattee in der Modifikation nach SIERSBAEK-NIELSEN (4) unter Verwendung von Dowex $1 \times 8 \quad 200-400$ mesh als Ionenaustauscher bestimmt. Für jeden Bestimmungstag wurde eine volle Standardkurve mit 5 Meßpunkten als Doppeliverte festgelegt. Gleichbleibende Mengen an Ionenaustauscher hatten sich zum Erreichen reproduzierbarer Werte als äußerst wichtig erwiesen. Durch Herstellen eines entsprechenden kleinen Meßbechers mit etwa $1 \mathrm{ml}$ Inhalt wurde diese Voraussetzung erfüllt. Eine optimale Steigung der Standardkurve wurde mit $12 \mathrm{ml}$ Poolserum (anstelle von $15 \mathrm{~m} /$ nach MURPHY) pro $500 \mathrm{ml}$ der ${ }^{125} \mathrm{~J}$-Thyroxin-TBG ${ }^{4}$ )-Lösung crzielt. Große Mengen dieses speziellen Poolserums wurden eingefroren. Um einen leicht bogenförmigen Verlauf der Standardkurve im unteren Meßbereich infolge fehlender Sättigung des TBG für diesen Bereich auszugleichen, wurde der Lösung nichtradioaktives Thyroxin zugesetzt. Mit $0,33 \mu \mathrm{g} \mathrm{T} / 500 \mathrm{~m} l$ Lösung konnte ein fast gradliniger Kurvenverlauf erreicht werden. - Das radioaktive ${ }^{125} \mathrm{~J}$-Thyroxin für das $\mathrm{T}_{4}-\mathrm{M}$ wurde mit einer spezifischen Aktivität von etwa $50 \mathrm{mC} / \mathrm{mg}$ und einer Reinheit von etwa $70-80 \%$ rom Radiochemical Center, Amersham, bezogen. Nach einer (zur Bestimmung von freiem Thyroxin notwendigen) Reinigungsdialyse wurden etwa $45-50 \mu \mathrm{C}$ zur Herstellung von $500 \mathrm{ml}$ der ${ }^{125} \mathrm{~J}-\mathrm{T}_{4}$-TBG-Lösung eingesetzt. $1 \mathrm{~m} l$ dieser Lösung ergab dann bei einer Zählausbeute von etwa $30-35 \%$ für ${ }^{125} \mathrm{~J}$ in dem verwendeten Philips-Meßgerät etwa $50000 \mathrm{Imp}$./Min.

Das $T_{4}-T$. (Tetrasorb-Abbott) wurde entsprechend der Vorschrift der Herstellerfirma bestimmt, wobei die korrekte Lage der Standardkurre durch Messung von 4 Punkten dieser Kurve (Einzelwerte) jeweils überprüft wurde. Angaben über die $\mathrm{Zu}$ sammensetzung der ${ }^{125} \mathrm{~J}-\mathrm{T}_{4}$-TBG-Lösung wurden vọ̣ der Herstellerfirma nicht gemacht.

Wie erwähnt, ist das Prinzip der $\mathrm{T}_{4}-\mathrm{M}$ - und $\mathrm{T}_{4}$-T-Methode gleich. Wesentliche Unterschiede bestehen aber in der Art, das TBGgebundene Thyroxin rom freien $T_{4}$ zu trennen: beim $T_{4}-M$ wird nach 18 minütiger Inkubation das vorhandene freie $T_{4}$ mittels des Ionenaustauschers sofort abgefangen und abzentrifugiert; gemessen wird das TBG-gebundene radioaktive $T_{4}$. Beim $T_{4}-T$ ist der Anionenaustauscher in einem Kunstharzschwamm enthalten. Nach 1stdg. Inkubation wird der TBG-gebundene Anteil der Radioaktivität abgesaugt und das am Schwamm verbleibende freie ${ }^{125} \mathrm{~J}-\mathrm{T}_{4}$ gemessen und als Prozentwert der Gesamtaktivität vor dem Absaugen angegeben. Zweimaliges Messen sämtlicher Proben vor und nach der Entfernung des TBG-gebundenen Anteils wird von der Herstellerfirma empfohlen.

Alle angegebenen Werte sind sowohl beim $\mathrm{T}_{4}-\mathrm{M}$ als auch $\mathrm{T}_{4}-\mathrm{T}$ hinsichtlich der Wiederfindung bei der Extraktion des Serum (83\% bzw. $80 \%$ ) korrigiert. Extraktion des $\mathrm{T}_{4}$ aus dem Serum erfolgte beim $\mathrm{T}_{4}-\mathrm{M}$ durch Petroläther-vergällten 95 proz. Alkohol, beim $\mathrm{T}_{4}-\mathrm{T}$ durch reinen 95 proz. Alkohol.

Zur Prüfung der Richtigkeit wurde nichtradioaktives Thyroxin zu je $20 \mathrm{~m} l$ eines Serums mit niedrigem Thyroxingehalt zugesetzt, so $\mathrm{da} ß$ sich der Thyroxin-Gehalt in diesem Serum um 1, 2, 3, 4, 6, 10, $14,18,22$ und $26 \mu \mathrm{g} / 100 \mathrm{ml}$ erhöhte. Kommerzielles Trockenplasma erwies sich nach Aufnahme in der vorgeschriebenen Menge an destilliertem Wasser als geeignete Ausgangslösung. Bei der Berechnung des Zusatzes an "kaltem" $T_{4}$ ist der 10 proz. Gewichtsanteil des verwendeten Natriumpentahydrates des LThyroxins berücksichtigt worden. Aus den so gewonnenen Seren mit definiertem Thyroxinzusatz wurden mit jeder der 3 Methoden Dreifachbestimmungen durchgeführt und die gemessenen Werte gegenüber dem erwarteten theoretischen Wert graphisch dargestellt.

Die Genauigkeit der Methoden wurde anhand eines EuthyreosePoolserums sowohl „von Tag zu Tag“ als auch ,,in Serie an einem Tag“ überprüft. Gleichzeitig wurde die Präzision von Doppelwerten in den einzelnen Meßbereichen mit Hilfe der Formel

ermittelt.

$$
s=\sqrt{\frac{\sum\left(\mathrm{x}_{1}-\mathrm{x}_{2}\right)^{2}}{\mathrm{~N}-1}}
$$

Zur Uberprüfung der Anvendbarkeit in der klinischen Routinediagnostik wurden die mit den drei Methoden simultan gewonnenen Ergebnisse aus 179 Patientenseren ausgewertet. Die Beurteilung des jeweiligen Stoffwechselstatus erfolgte in der Endokrinologischen Ambulanz und im Institut für Nuklearmedizin außer durch die $\mathrm{T}_{4}$-Bestimmung noch durch das klinische Bild, andere Serumparameter (Cholesterin, Enzymaktivitäten), Radiojodtest mit PB $\left.^{131} 1^{4}\right)-32$ Stundenwert. Wenn erforderlich, wurde die Diagnose durch $\left.\mathrm{T}_{3}\right)^{4}$ - in vitro-Test und Bestimmung des freien Thyroxins sowie des Grundumsatzes weiter gesichert.

\section{Ergebnisse}

\section{Präzision}

Aus Tabelle 1 sind Daten für die Genauigkeit der mit den 3 Methoden gemessenen Werte zu ersehen. Als Maß der Präzision von Mehrfachbestimmungen an einem Tag

Tab. 1

Präzision dreier Methoden zur Bestimmung der Thyroxinkonzentration im Serum

\begin{tabular}{lccc}
\multicolumn{1}{c}{ Methode } & $\mathrm{T}_{4}-\mathrm{PBI}$ & $\mathrm{T}_{4}-\mathrm{M}$ & $\mathrm{T}_{4}-\mathrm{T}$ \\
\hline Präzision in Serie & 3,5 & 7,7 & 15,8 \\
(VK in \%) & $(10)$ & $(10)$ & $(10)$ \\
Präzision von Tag zu Tag & 3,1 & 7,1 & 11,6 \\
(VK in \%) & $(20)$ & $(24)$ & $(10)$ \\
\hline
\end{tabular}

Präzisionsmaß von Doppelwerten (s)

\begin{tabular}{|c|c|c|c|c|}
\hline \multirow{3}{*}{ 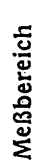 } & $0-10 \mu \mathrm{g} / 100 \mathrm{ml}$ & $\pm \underset{(4)}{0,20}$ & $\pm \underset{(4)}{0,55}$ & $\begin{array}{c} \pm 0,68 \\
\text { (4) }\end{array}$ \\
\hline & $11-20 \mu \mathrm{g} / 100 \mathrm{ml}$ & $\pm \underset{(3)}{0,61}$ & $\pm \underset{(3)}{0,67}$ & $\begin{array}{l} \pm 2,10 \\
(3)\end{array}$ \\
\hline & $21-30 \mu \mathrm{g} / 100 \mathrm{ml}$ & $\pm \underset{(3)}{1,01}$ & $\pm 1,50$ & $\begin{array}{c} \pm 3,68 \\
(3)\end{array}$ \\
\hline
\end{tabular}

(Präzision in Serie) und an verschiedenen Tagen über 1 bis 3 Monate (Präzision von Tag zu Tag) wurde der Variationskoeffizient ermittelt; als Präzisionsmaß von Doppelwerten wurde die Standardabweichung der Doppelwerte in verschiedenen Meßbereichen angegeben. - Aus der Tabelle geht hervor, daß bei Mehrfachbestimmungen die Berechnung des Gesamtthyroxins aus dem PBI die geringste Streuung der Einzelwerte um den Mittelwert aufweist. Eine etwa doppelt so hohe Streuung ergibt sich aus der Isotopenverdrängungsmethode von MURPHY und PATTEE, während beim $\mathrm{T}_{4}$ - $\mathrm{T}$ die Streuung etwa 4 bis 5 fach höher als beim $\mathrm{T}_{4}$-PBI liegt. Bei der Bestimmung der Standardabweichung von Doppelwerten in den einzelnen $\mathrm{MeB}$ bereichen zeigt sich, daß die Genauigkeit mit allen Methoden um so stärker abnimmt, je höher die bestimmten Thyroxinwerte sind. Während aber für das $T_{4}-\mathrm{PBI}$ und $\mathrm{T}_{4}-\mathrm{M}$ in allen 3 Bereichen vergleichbare Präzisionswerte gefunden wurden, liegt die Genauigkeit der Doppelwerte für das $\mathrm{T}_{4}$-T im Bereich 11 bis $20 \mu \mathrm{g} /$ $100 \mathrm{~m} l$ und 21 bis $30 \mu \mathrm{g} / 100 \mathrm{~m} l$ um etwa das Dreifache unter dem der beiden anderen Methoden.

\section{Richtigkeit}

Zur Ubberprüfung, um wieviel in den 3 Methoden ein einzelner Meßwert von der tatsächlich im Serum vorhandenen Thyroxinmenge abweicht, wurden bekannte 
Abb. 1

Werte zur Richtigkeit von Methoden zur Thyroxinbestimmung; Abscisse: eingesetzte Thyroxinkonzentration; Ordinate: gemessene Thyroxinkonzentration

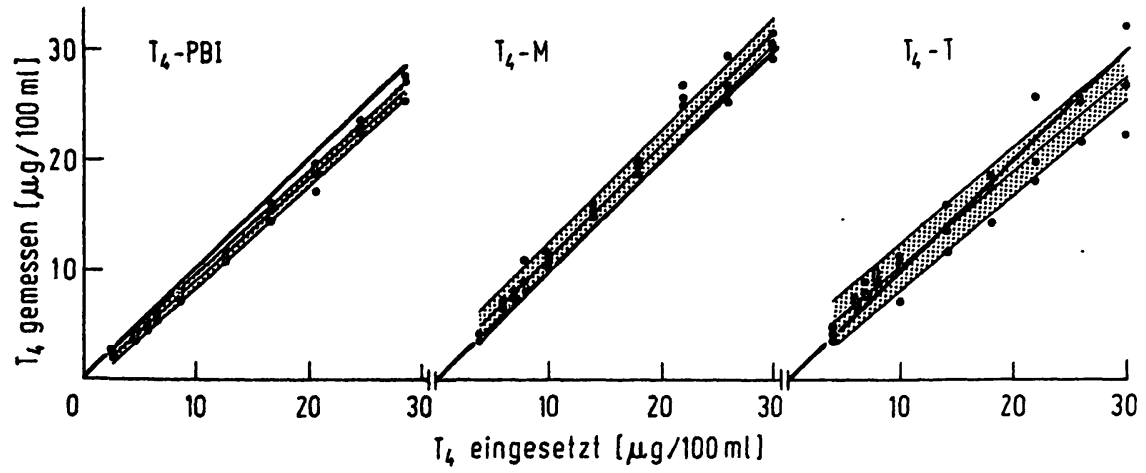

Mengen an Thyroxin zugesetzt und die beobachteten Resultate den erwarteten Werten gegenübergestellt (s. Abb. 1). Als Maß für die Übereinstimmung bzw. Nichtübereinstimmung der Erwartung mit der Beobachtung dienten neben dem Korrelationskoeffizienten die statistische Prüfung, ob die individuellen Charakteristika der 3 Regressionsgeraden von denen der Idealregression $y=0+1 x$ signifikant differieren. In Tabelle 2 sind die individuellen Daten der 3 Regressionsgeraden und der Signifikanzprüfung zusammengestellt.

Tab. 2

Daten zur Beurteilung der Richtigkeit von Methoden der Thyroxinmessung im Serum

\begin{tabular}{|c|c|c|c|}
\hline & $T_{4}-\mathrm{PBI}$ & $T_{4}-M$ & $T,-T$ \\
\hline $\mathbf{r}$ & 0,9972 & 0,9894 & 0,9632 \\
\hline$a$ & $-0,3775$ & 0,7798 & 1,6538 \\
\hline b & 0,9471 & 1,0436 & 0,8664 \\
\hline sy $\cdot x$ & 0,6246 & 1,3685 & 2,1598 \\
\hline$t=\frac{b-1}{s b}$ & $2 P \stackrel{4,000}{<}<0,001$ & $\begin{array}{c}1,6432 \\
0,20>2 P>0,10\end{array}$ & $\begin{array}{c}2,9301 \\
0,01>2 P>0,005\end{array}$ \\
\hline$t=\frac{a-0}{S a}$ & $0,10>2 \mathrm{P}>0,05$ & $\begin{array}{c}1,5953 \\
0,20>2 \mathrm{P}>0,10\end{array}$ & $\begin{array}{c}2,1511 \\
0,05>2 P>0,025\end{array}$ \\
\hline
\end{tabular}

Aus Abbildung 1 und Tabelle 2 ist $z u$ ersehen, daß sich beim $\mathrm{T}_{4}$-PBI eine sehr gute Übereinstimmung $z$ wischen den erwarteten und den gemessenen Werten ergab. Der Schnittpunkt der Regressionsgeraden mit der y-Achse liegt im negativen Bereich, wobei der Unterschied zum Idealwert $\mathrm{a}=0$ aber nicht statistisch gesichert ist. Die geringe Abweichung der Steigung $b$ von 1 läßt sich sichern, da die Streuung um die Regression sehr klein ist. Es geht hieraus hervor, daß bei der Errechnung des $\mathrm{T}_{4}$ aus dem PBI gewöhnlich etwas zu niedrige Werte erhalten werden.

Beim $\mathrm{T}_{\mathbf{4}}-\mathrm{M}$ liegt ebenfalls eine gute Korrelation vor; der Schnittpunkt der Geraden liegt auf dem positiven Schenkel der $y$-Achse; $b$ ist etwas größer als 1 . Demnach wird man mit der MurpHy-Methode eher etwas zu hohe Werte finden. Obwohl sich b und a von den Idealwerten stärker unterscheiden, sind diese Differenzen infolge der größeren Streuung nicht $z u$ sichern.

Der Korrelationskoeffizient von 0,963 beim $\mathrm{T}_{4}-\mathrm{T}$ ist deutlich niedriger als der der anderen Methoden, die Streuung um die Regression ist gegenüber dem $\mathrm{T}_{4}$-PBI dreifach höher; durch entsprechend signifikant erhöhten Wert von a und signifikant erniedrigten Wert von $b$ weicht die Regressionsgerade in ihrem Verlauf erheblich von der 1:1-Beziehung ab. Hervorzuheben ist, daß sich mit dieser Methode wie mit der ihr entsprechenden Murphy-Methode im unteren Meßbereich etwas zu hohe Werte ergeben, während ab $15 \mu \mathrm{g} / 100 \mathrm{~m} l$ häufig zu niedrige Ergebnisse auftreten.

Aus Abbildung 1 geht weiterhin hervor, daß mit dem $\mathrm{T}_{4}$-PBI bis in die höchsten hier eingesetzten Bereiche verläßliche Werte mit geringer Streuung gemessen werden können. Die Grenze der Verläßlichkeit liegt beim $\mathrm{T}_{4}$-M bei etwa $20 \mu \mathrm{g} / 100 \mathrm{ml}$, beim $\mathrm{T}_{4}$ - $\mathrm{T}$ scheint sie nach diesem Versuch bereits wenig oberhalb von $10 \mu \mathrm{g} /$ $100 \mathrm{ml}$ erreicht $\mathrm{zu}$ sein.

Die Anwendbarkeit der 3 Methoden in der klinischen Routinediagnostik wurde an Seren von 131 euthyreoten, 33 hyperthyreoten und 15 hypothyreoten Patienten geprüft; mit jeder Methode wurde in jedem Serum ein Wert bestimmt. Die Mittelwerte für die einzelnen Bereiche sind in Tabelle 3 dargestellt. Es fällt auf,

Tab. 3

Mittelwerte der Thyroxinkonzentration $(\mu \mathrm{g} / 100 \mathrm{ml})$ in Seren von eu-, hypo- und hyperthyreoten Patienten, gemessen mit 3 verschiedenen Methoden

\begin{tabular}{lccc}
\hline & $T_{-}-P_{1}$ & $T_{4}-M$ & $T_{4}-T$ \\
\hline $\begin{array}{l}\text { Hypothyreose } \\
(n=15)\end{array}$ & $3,04 \pm 2,23$ & $2,65 \pm 1,00$ & $3,25 \pm 1,51$ \\
$\begin{array}{l}\text { Euthyreose } \\
(n=131)\end{array}$ & $10,34 \pm 4,53$ & $9,33 \pm 2,33$ & $8,94 \pm 2,42$ \\
$\begin{array}{l}\text { Hyperthyreose } \\
(n=33)\end{array}$ & $19,88 \pm 4,19$ & $17,52 \pm 3,38$ & $16,12 \pm 3,28$ \\
\end{tabular}

daß in diesem Falle die $\mathrm{T}_{\mathbf{4}}$-PBI-Ergebnisse nicht $\mathrm{zu}$ tief, wie nach Abbildung $1 \mathrm{zu}$ erwarten wäre, liegen, sondern höher als die entsprechenden $T_{4}-M$ - und $T_{4}-T$-Werte. Weiterhin ist zu erwähnen, daß der niedrigste Mittelwert im Hyperthyreosebereich mit dem $\mathrm{T}_{4}-\mathrm{T}$ ermittelt wurde. Die Ursache für die relativ hohen $T_{4}-\mathrm{PBI}-$ Mittelwerte liegt in der Jodkontamination einzelner Seren, die zu Fehlbestimmungen mit dieser Methode führte. Durch Vergleich mit den Ergebnissen der beiden anderen, absolut spezifischen Methoden konnten diese kontaminierten Proben ermittelt werden. Die Seren, in denen der $\mathrm{T}_{4}$-PBI-Wert im Hypo- und Euthyreosebereich um mehr 


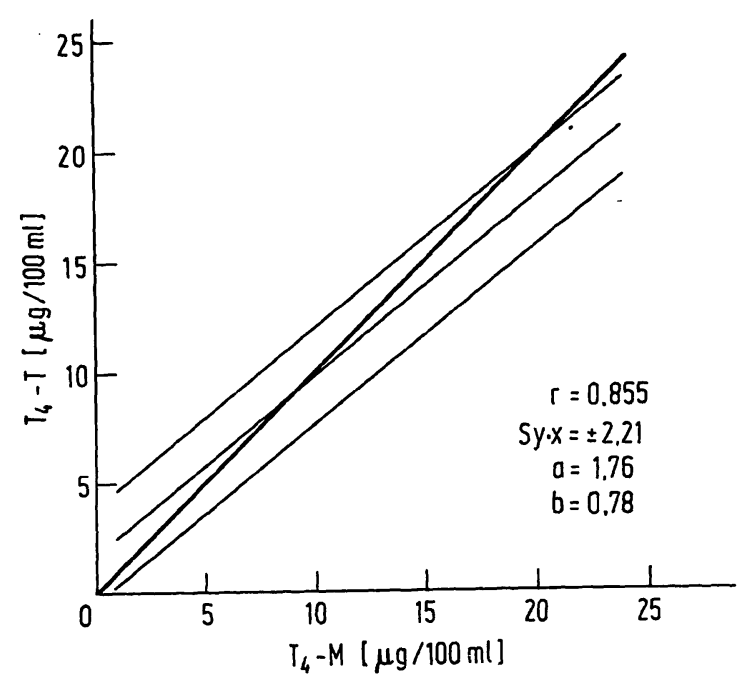

Abb. 2

Daten zur Konzentration von Thyroxin im Serum, gemessen mit den Methoden $T_{4}-M$ (Abscisse) und $T_{4}-T$ (Ordinate)

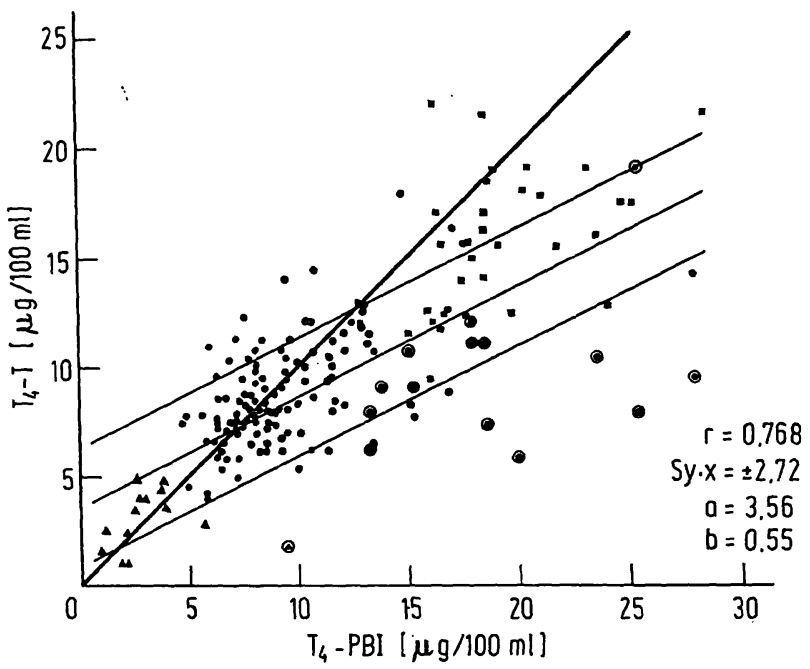

Abb. 4

Daten zur Thyroxinkonzentration im Serum, gemessen mit den Methoden $T_{4}-P B I$ (Abscisse) und $T_{4}-T$ (Ordinate)

$\odot$ kontaminierte Seren; Hyperthyreose Hypothyreose Euthyreose;

als $4 \mu \mathrm{g} / 100 \mathrm{~m} l$, im Hyperthyreosebereich um mehr als $8 \mu \mathrm{g} / 100 \mathrm{~m} l$ gegenüber beiden anderen Ergebnissen lag, wurden als kontaminiert angesehen. 15 Proben $=8,4 \%$ des Gesamtkollektivs erfüllten diese Bedingungen!

In den Abbildungen 2, 3 und 4 sind die Beziehungen der 3 Methoden zueinander im Gesamtkollektiv durch die entsprechenden Regressionsgeraden und Korrelationskoeffizienten dargestellt. Die beste Übereinstimmung mit dem höchsten Korrelationskoeffizienten $(r=0,855)$, dem größten Regressionskoeffizienten, der geringsten Streuung um die Regressionsgerade und dem niedrigsten Wert von a ergab sich beim Vergleich der beiden Isotopenverdrängungsmethoden (Abb. 2).

Die mangelhafte Spezifität der Jodbestimmung für die Messung der Thyroxinkonzentration beeinflußt den Vergleich zwischen $\mathrm{T}_{4}-\mathrm{PBI}$ und $\mathrm{T}_{4}-\mathrm{M}$ bzw. $\mathrm{T}_{4}-\mathrm{T}$ (Abb. 3 und 4) entscheidend. Die 15 jodkontaminierten Seren

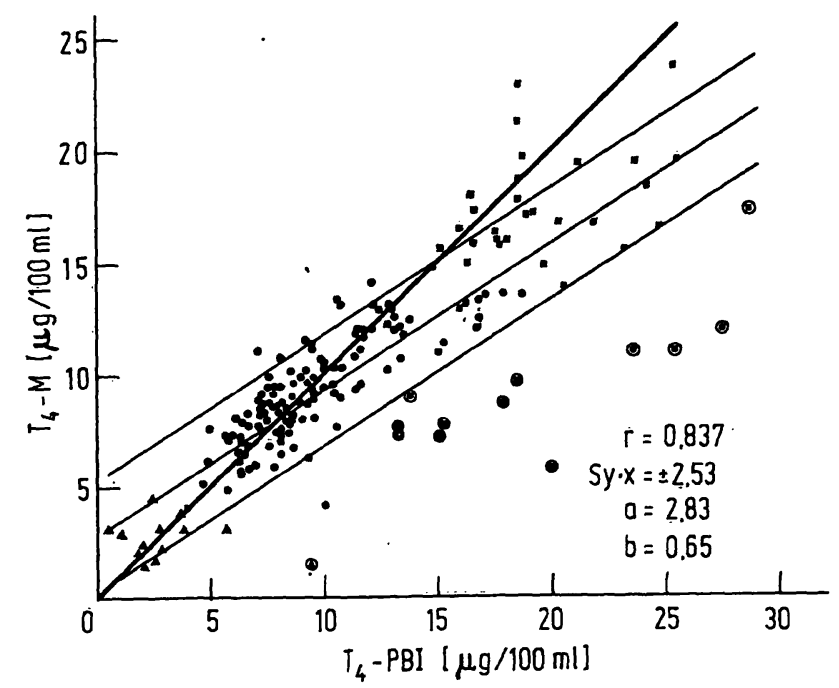

Abb. 3

Daten zur Thyroxinkonzentration im Serum, gemessen mit den $\mathrm{Me}$ thoden $\mathrm{T}_{4}-\mathrm{PBI}$ (Abscisse) und $\mathrm{T}_{4}-M$ (Ordinate)

$\odot$ kontaminierte Seren; Hyperthyreose - Euthyreose;
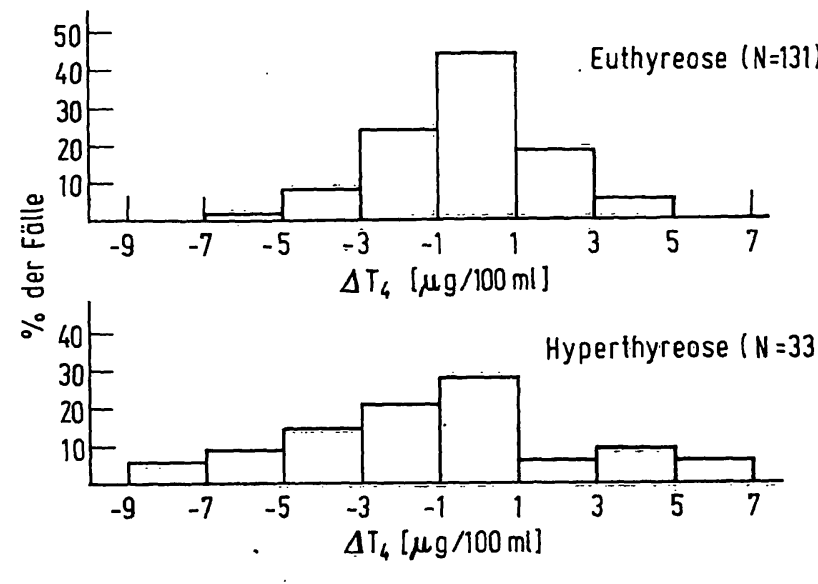

Abb. 5

Differenzen der Thyroxinkonzentrationen ( $\left.T_{-}-T_{\text {minus }} T_{4}-M\right)$, gruppiert und in \% der Zahl der in der Euthyreose- bzw. Hyper-

führten zu einer erheblichen Abweichung der Regressionsgeraden von der 1:1-Beziehung. Bei der Regression $\mathrm{T}_{4}-\mathrm{T}$ auf $\mathrm{T}_{4}$-PBI bewirkten die relativ geringe Präzision und Genauigkeit der $\mathrm{T}_{4}$-T-Methode eine weitere Reduktion der Korrelation.

Als letztes wurde untersucht, ob für die relativ schlechte Übereinstimmung der beiden spezifischen Methoden in bestimmten Meßbereichen durch Analyse der in der klinischen Routine erhaltenen Ergebnisse eine Ursache zu finden war. $\mathrm{Zu}$ diesem Zweck wurde in Abbildung 5 die Größe der Differenz zwischen den jeweiligen Einzelmessungen $\left(T_{4}-T\right.$ minus $\left.T_{4}-M\right)$ ermittelt, gruppiert und in Prozent der Anzahl dargestellt. Im euthyreoten Bereich unterscheiden sich $86 \%$ der Fälle um $\pm 3 \mu \mathrm{g} / 100 \mathrm{ml}$. Im hyperthyreoten Bereich war die Treppenkurve aber weit nach links verschoben, d. h., daß hier in der überwiegenden Mehrzahl der Fälle mit der $\mathrm{T}_{4}$-T-Methode niedri- 
gere Werte als mit dem $\mathrm{T}_{4}-\mathrm{M}$ gemessen wurde. Für den hypothyreoten Bereich werden durchschnittlich etwas zu hohe Werte mit dem $\mathrm{T}_{4}$ - $\mathrm{T}$ angegeben. Ähnliche Verhältnisse ergaben sich beim entsprechenden Vergleich zwischen dem $T_{4}-P B I$ und $T_{4}-T$, allerdings waren hierbei die Unterschiede durch den Einfluß der jodkontaminierten Seren noch prononcierter.

\section{Diskussion}

Die Bestimmung des proteingebundenen Jods bietet den entscheidenden Vorteil eines vollautomatisierten Verfahrens. Etwa 120 Proben können unter den bei uns üblichen Kautelen (Screening zur Erfassung stark jodkontaminierter Proben, Geschwindigkeit von 20 Proben pro Stunde) in etwa 8 Stunden gemessen werden. Die Präzision der PB127I-Bestimmung ist in allen Meßbereichen sehr gut. Die Prüfung der Richtigkeit der Methode zeigte, daß etwas zu niedrige Werte gemessen werden. Nach Beobachtungen in unserem Labor ist erwiesen, daß bei Behandlung des zu messenden Serums mit Amberlite IRA-401 als Ionenaustauscher, nicht nur Jodid dem Serum entzogen wird, sondern auch etwa 5 bis $10 \%$ des Thyroxinjodes (5). Bei Analyse der Abbildung 1 ist ersichtlich, daß die Regressionsgerade fast genau um diesen Betrag von der Idealfunktion abweicht. Um die große Probenkapazität der Apparatur voll auszuschöpfen, lohnt sich die Bestimmung des $\mathrm{PB}^{127} \mathrm{I}$ in großen Krankenhäusern mit entsprechender endokrinologischer Abteilung. Der gravierende Nacbteil der Methode liegt in ihrer mangelbaften Spezifität. Immerhin hätten sich in unserem Kollektiv bei alleiniger Diagnostik durch die PBI-Bestimmung 15 klinisch-chemische Fehldiagnosen $=8,4 \%$ ergeben. 14 tatsächlich euthyreote Patienten wären als hyperthyreot, 1 hypothyreoter $\mathrm{Pa}$ tient als euthyreot angesehen worden.

Die Murphey-Pattee-Methode ist hinsichtlich der Präzision der PBI-Bestimmung eindeutig unterlegen. Die Streuung um den Mittelwert als $\mathrm{Ma}$ der Präzision lag bei wiederholten Bestimmungen eines Euthyreose-Poolserums mit $7,7 \mu \mathrm{g} / 100 \mathrm{~m} l \pm 0,6$ im gleichen Bereich wie von anderen Autoren mitgeteilt (MURPHY (3): 7,2 $\mu \mathrm{g} /$ $100 \mathrm{ml} \pm 0,7$, Siersbaek-NieLsEN (4): $8,0 \pm 0,9$, Arango (6): $7,4 \pm 0,4$ ). Für den hyperthyreoten Bereich werden auch von diesen Autoren deutlich größere Standardabweichungen angegeben. Die Richtigkeit der Murphy-Patree-Methode erfüllt mit einem Korrelationskoeffizienten von $r=0,989$ die üblichen Anforderungen an ein klinisch-chemisches Verfahren. Thre Bestimmungskapazität liegt in dem von uns ermittelten Ablauf bei 100 Seren/5 Stunden, entspricht also etwa der Leistungsfähigkeit der PBI-Bestimmung. Allerdings erfordert diese hohe Anzahl konzentriertes und sorgfältiges Arbeiten während der gesamten Zeit. Erreicht werden kann diese Kapazität weiterhin nur durch Konstruktion einer leistungsfähigen Abdampfvorrichtung (48 Proben) und durch weitgehenden Gebrauch halbautomatischer, hinreichend genauer Pipetten (Reproduzierbarkeit besser als 0,5\%). Genaue Kenntnis der Fehlermöglichkeiten und Erfahrungen im Umgang mit der zu verwendenden Radioaktivität sind weitere Voraussetzungen für einen befriedigenden Einsatz der Murphy-PATTEE-Methode. Der in unserem Patientenkollektiv zwischen $T_{4}$-PBI und dem $\mathrm{T}_{4}-\mathrm{M}$ errechnete Korrelationskoeffizient von 0,837 stimmt fast genau mit einer von MURPHY (7) anhand von 300 Vergleichsbestimmungen ermittelten Korrelation von $r=0,823$ überein. Unter diesen 300 Fällen befanden sich 40 jodkontaminierte $(=13,3 \%$ ) Seren, die in die Berechnungen eingingen.

Hinsichtlich der Beurteilung der Anwendbarkeit der Murphy-Pattee-Methode gilt im Prinzip das gleiche, wie für die PBI-Bestimmung: Nur für Laboratorien mit großem Probenanfall ist der Aufbau dieser Methode lohnend, aber wegen der hohen Spezifität für Thyroxin und des gegenüber dem kommerziellen TetrasorbTest mindestens $40-$ bis 50 fach niedrigeren finanziellen Aufwandes für eine Bestimmung auch empfehlenswert.

Der kommerzielle Tetrasorb-Test erwies sich in dieser Untersuchung sowohl im standardisierten Testprogramm als auch bei der Anwendung in praxi hinsichtlich Präzision und Richtigkeit den beiden anderen Tests unterlegen. Allerdings muß erwähnt werden, daß die Methode von uns auch in Bereichen verglichen wurde, in denen nach Empfehlung der Herstellerfirma Angaben über einen präzisen Wert unterbleiben sollten. Die Standardkurve des Tests verläuft zwischen 0 und $12 \mu \mathrm{g} /$ $100 \mathrm{~m} l$ fast geradlinig, oberhalb dieses Wertes flacht sie aber immer mehr ab und zeigt oberhalb von $20 \mu \mathrm{g} /$ $100 \mathrm{~m} l$ nur noch eine sehr geringe Steigung, so daß Werte über $20 \mu \mathrm{g} / 100 \mathrm{~m} l$ prinzipiell nicht abgelesen werden sollten. Gegenüber der Murphy-Methode verläuft die standardisierte Stammkurve des TetrasorbTests insgesamt aber sehr viel flacher. Die mangelnde Präzision der Methode könnte auf die fehlende Steilheit der Stammkurve zurückgeführt werden, bei der geringe Unterschiede in den gemessenen counts zu größeren Differenzen in den abgelesenen Ergebnissen Anlaß geben. Neben diesem vielleicht methodischen Fehler können natürlich Ungenauigkeiten bei der technischen Durchführung das Vergleichsergebnis zuungunsten der $\mathrm{T}_{4}$-T-Methode verschoben haben, zumal von anderen Autoren sehr gute Korrelationskoeffizienten bei Vergleich zwischen $\mathrm{T}_{4}$-PBI und $\mathrm{T}_{4}$ - $\mathrm{T}$ mitgeteilt werden. So ermittelten KENNEDY und ABELson (8) einen Korrelationskoeffizienten von $r=0,92$ bei Vergleich von 34 Fällen, Kaplan (9) ein $r$ von 0,88 , wobei keine Angabe über die Fallzahl gemacht wurde. Nähere Einzelheiten über Meßbereiche und Einschluß jodkontaminierter Seren in die Berechnung dieser Korrelationen waren nicht zu ersehen.

Die maximale Kapazität der $\mathrm{T}_{4}$-T-Methode liegt bei etwa 40 Proben/5 Stunden. Bedingt durch die lange Inkubationszeit von 1 Stunde und das zweimalige Zählen jeder Probe sowie durch die relativ umständliche mitgelieferte Abdampfvorrichtung läßt sich die Kapazität der beiden anderen Methoden nicht erreichen. Ein Nachteil der $\mathrm{T}_{4}$-T-Methode ist auch der hohe Preis, der 
in keinem Verhältnis zu den Kosten der beiden anderen Verfahren steht.

Der Vorteil des kommerziellen Testes liegt in der von der Firma bereits standardisierten Handhabung. Alle Geräte, Lösungen, Reagenzien und die fertige Standardkurve können kommerziell bezogen werden. Zur Ausführung des Tests ist praktisch nur ein entsprechendes
Zählgerät erforderlich. Aus diesem Grunde ist der Test für Laboratorien mit geringem Probenanfall oder solchen, in denen Thyroxinbestimmungen nur sporadisch erforderlich werden, geeignet, wenn die oben gegebenen Hinweise auf die Präzisionsschwäche im hohen Thyroxinbereich steț bęrücksichtigt werden.

\section{Literatur}

1. Ekins, R. P., Clin. Chim. Acta, Amsterdam 5, 453 (1960). 2. Murphy, B. E. P. und C. J. Pattee, J. Clin. Endocrin., Springfield 24, 187 (1964). - 3. Murphy, B. E. P. und C. JACHAN, J. Laborat. Clin. Med. S. Louis 66, 161 (1965). - 4. SiersbaekNielsen, K., Acta med. scand. 181, 327 (1967). - 5. KöDding, R. und H. L. KrüskeMPER, diese Z. 8, 425 (1970). - 6. ArANGo, G.,
W. E. Mayberry, T. J. Hockert und L. R. Elvebaek, Mayo Clin. Proc. 43, 503 (1968). - 7. Murphy, B. E. P., C. J. Pattee und A. J. Gold, J. Clin. Endocrin., Springfield 26, 247 (1966). 8. Kennedy, J. A. und D. M. Abelson, J. Clin. Path. London 20, 89 (1967). - 9. KapLAN, B. C., persönl. Mitteil. an Abbott-Laboratories (1967).
Anschrift $\left.{ }^{1}\right)$ :

Abt. Klin. Endokrinologie

Dept. Innere Medizin 3000 Hannover

Podbielskistr. 380 